\section{História prévia de realização de teste de Papanicolaou e câncer do colo do útero: estudo caso-controle na Baixada Fluminense, Rio de Janeiro, Brasil}

\author{
Previous history of Pap smears and cervical cancer: \\ a case-control study in the Baixada Fluminense, \\ Rio de Janeiro State, Brazil
}

\author{
1 Hospital Geral de Nova \\ Iguaçu, Nova Iguaçu, Brasil. \\ 2 Instituto de Medicina \\ Social, Universidade do \\ Estado do Rio de Janeiro, Rio \\ de Janeiro, Brasil. \\ 3 Escola Nacional de Saúde \\ Pública Sergio Arouca, \\ Fundação Oswaldo Cruz, Rio \\ de Janeiro, Brasil. \\ Correspondência \\ M. I. Nascimento \\ Hospital Geral de Nova \\ Iguaçu. \\ Av. Henrique Duque Estrada \\ Mayer 953, Nova Iguaçu, RJ \\ 26030-380, Brasil. \\ ysamaria@uol.com.br
}

\begin{abstract}
This hospital-based case-control study (20072010) aimed to estimate the association between previous Pap smear and cervical cancer diagnosis in the Baixada Fluminense, Rio de Janeiro State, Brazil. The sample consisted of 152 histologically confirmed cases and 169 controls from the same hospital as the cases. The analysis was conducted according to a model in three hierarchical levels considering sociodemographic characteristics (distal), sexual/reproductive and lifestyle factors (intermediate), and previous Pap smear (proximal). Odds ratios (OR) and respective 95\% confidence intervals (95\%CI) were calculated using unconditional logistic regression. History of three or more Pap smears was associated with an $84 \%$ reduction in cervical cancer risk $(O R=0.16$; 95\% CI: 0.074; 0.384) after adjusting for selected variables. The results highlight the importance of preventive gynecological examination for reducing the risk of cervical cancer and emphasize the need to increase adherence to the guidelines of the Brazilian program, besides identifying and scheduling women that have proven reluctant to submit to cervical cancer screening.
\end{abstract}

Uterine Cervical Neoplasms; Vaginal Smears; Health Services Accessibility
Maria Isabel do Nascimento 1

Gulnar Azevedo e Silva 2

Gina Torres Rego Monteiro ${ }^{3}$

\section{Introdução}

Na maior parte do mundo, o teste de Papanicolaou ainda é a principal ferramenta para o rastreamento de massa das lesões cervicais, embora, atualmente, haja outras tecnologias disponíveis ${ }^{1}$. O exame consiste numa sequência de etapas laboratoriais que ao final permite identificar, nas células esfoliadas do colo uterino, alterações suspeitas de transformação neoplásica 2 . Apesar de o teste de Papanicolaou estar em uso para prevenção do câncer do colo do útero há mais de 50 anos, a doença ainda responde por metade da carga global de câncer ginecológico no mundo, fato atribuído à falta de efetivo programa de rastreamento nos países de média e baixa renda 3 .

Alguns estudos observacionais têm investigado a efetividade da citologia convencional na redução de risco de câncer do colo de útero 4,5,6,7,8. Com políticas de rastreamento baseadas em coletas citológicas a cada três anos e faixa etária de 25-64 anos, pode-se alcançar até 90\% de redução na incidência da doença, supondo que o protocolo tenha sido rigorosamente cumprido 9 . Ainda assim, no contexto de programas de saúde pública e de sistemas de saúde específicos é importante quantificar a magnitude de tal benefício 7 .

No Brasil, com a implantação na década de 1990 do Programa Viva Mulher, foram ampliados os recursos para o controle desse câncer, permanecendo o protocolo de rastreamento do tipo oportunístico e com o uso do teste de Papanico- 
laou ${ }^{10}$. Em relação à periodicidade, a recomendação é que o exame seja repetido a cada três anos após a realização de dois exames consecutivos com intervalo de um ano e com resultados negativos. Embora os resultados de inquéritos recentemente conduzidos sugiram que a proporção de mulheres da população geral que faz uso do exame esteja aumentando no país 11, o efeito do teste de Papanicolaou na redução de risco de câncer do colo de útero foi mensurado pela investigação de casos da doença detectados na cidade de São Paulo 12 antes da implantação das ações do programa brasileiro.

Na Baixada Fluminense - área integrante da Região Metropolitana do Rio de Janeiro - o teste de Papanicolaou tem sido disponibilizado na rede pública de saúde, além do que a prevalência da infecção pelo HPV parece ser mais baixa do que as que têm sido estimadas em outras regiões do país 13. Contudo, verifica-se uma ocorrência contínua de casos novos de câncer do colo de útero nos municípios da região (DATASUS. Sistema de informações do câncer do colo do útero. http://tabnet.datasus.gov.br/CGI/tabcgi. exe?siscolo/DEFtodos/RJHCOLO.def, acessado em 15/Nov/2010) e não se conhece qual a contribuição do teste de Papanicolaou no desenvolvimento de tais casos. Ante tal questão é importante considerar, primeiramente, que a história natural do câncer do colo de útero envolve um processo dinâmico de longa duração favorecendo a detecção de doença na fase intraepitelial 14 . Adicionalmente, os sintomas relacionados ao tumor invasivo determinam a busca pelo exame 15 . Uma estratégia para lidar com essa situação é a exclusão de testes realizados durante um determinado período de tempo, de modo que a análise se restrinja à fase assintomática de doença e/ou de verdadeira lesão precursora, detectável por exame citopatológico 16. Entendendo que a análise dos exames dos últimos 36 meses da entrevista/diagnóstico não permitiria adequadamente distinguir se eles foram motivados pela presença da doença, podendo, assim, incorrer no viés de causalidade reversa 17 , o objetivo deste estudo foi estimar o efeito da história prévia de realização do teste de Papanicolaou no desfecho câncer do colo de útero em mulheres residentes na Baixada Fluminense, considerando algumas características individuais e os exames realizados além dos 36 meses da entrevista/diagnóstico.

\section{Métodos}

\section{Delineamento e cenário do estudo}

Este estudo caso-controle hospitalar teve como desfecho os casos de câncer do colo de útero diagnosticados entre janeiro de 2007 e agosto de 2010, no Hospital Geral de Nova Iguaçu (HGNI), unidade integrante da rede do Sistema Único de Saúde (SUS). O hospital é referência para atendimento secundário de lesões precursoras do câncer do colo de útero rastreadas em mulheres residentes na Baixada Fluminense pelo programa brasileiro de prevenção e controle dessa neoplasia.

O território de abrangência Regional Baixada Fluminense é composto por 12 municípios (Belford Roxo, Duque de Caxias, Itaguaí, Japeri, Magé, Mesquita, Nilópolis, Nova Iguaçu, Queimados, São João de Meriti, Paracambi e Seropédica), com população estimada em 1.200.000 mulheres em idade fértil (DATASUS. Indicadores de saúde. http://tabnet.datasus.gov.br/tabdata/cadernos/ rj.htm, acessado em 15/Nov/2010). Em 2000, o Índice de Desenvolvimento Humano (IDH-M) dos municípios da região estava situado na faixa média (variação de 0,677 em Mesquita a 0,788 em Nilópolis), sugerindo condições socioeconômicas menos favorecidas em relação ao Rio de Janeiro, capital do Estado (IDH-M 0,842) 18.

O estudo foi desenvolvido sob a coordenação da Escola Nacional de Saúde Pública Sergio Arouca, Fundação Oswaldo Cruz (ENSP/FIOCRUZ), como parte da tese de doutoramento de uma das autoras (M. I. N.), realizada no Programa de Pósgraduação em Saúde Pública e Meio Ambiente.

\section{Amostragem e população de estudo}

O tamanho da amostra foi calculado tendo em conta a precisão de $95 \%$, o poder estatístico de $80 \%$, a estimativa de risco relativo (OR - odds $\mathrm{ra}$ tio) de 2,0 e a relação de um controle por caso. Tendo em vista a prevalência de $33 \%$ de realização do teste de Papanicolaou no SUS, no Estado do Rio de Janeiro, período 2002-2003 19, foi prevista uma amostra de pelo menos 148 casos e 148 controles.

\section{Participantes}

Os critérios de inclusão das participantes do estudo foram: idade de 25-69 anos e tempo de residência em município da Baixada Fluminense não inferior a 36 meses.

Os casos foram mulheres com diagnóstico de câncer do colo de útero confirmado por histopatologia e codificado como C53 pela Classificação 
Internacional de Doenças (CID-10) 20, atendidas no período do estudo, no HGNI. Os critérios de exclusão de casos foram: história pregressa de câncer ginecológico, estágios terminais de câncer e problemas de saúde mental impeditivos de responder às perguntas do questionário.

Os controles foram mulheres acompanhantes de pacientes internados nas enfermarias de adultos no mesmo hospital. No HGNI, acompanhantes são as pessoas devidamente autorizadas a fornecer cuidados e permanecer ao lado do paciente durante a hospitalização. Os critérios de exclusão dos controles foram: (i) nunca ter tido relação sexual (mulheres virgens); (ii) antecedente de histerectomia; (iii) história pregressa de câncer ginecológico; e (iv) transtornos de saúde mental. Os critérios (i) e (ii) referem-se a grupos de mulheres em situações especiais que não são alvo do rastreamento pelo programa brasileiro 21; o terceiro critério foi estabelecido em função de o estudo ter tido enfoque nos casos incidentes; e possíveis dificuldades que poderiam impedir a compreensão de perguntas do questionário e, consequentemente suas respostas, determinaram o último critério de exclusão.

Os controles foram pareados aos casos segundo idade e município de residência. O pareamento foi feito pela frequência esperada de entrada de casos considerando cinco faixas etárias (25-29, 30-39, 40-49, 50-59 e 60-69 anos) combinadas com três classes de municípios da Baixada Fluminense agrupados segundo a proximidade geográfica entre eles, como segue: classe [i] Nova Iguaçu, Duque de Caxias e Magé; classe [ii] Queimados, Seropédica, Japeri, Paracambi e Itaguaí; classe [iii] Belford Roxo, Nilópolis, Mesquita e São João de Meriti.

A seleção das acompanhantes seguiu os critérios de inclusão e exclusão e a distribuição esperada de casos. Em dias alternados, visitava-se aleatoriamente, algum dos departamentos de pacientes adultos (Clínica Médica, Cirurgia Geral, Ortopedia, Ginecologia, Obstetrícia, Neurocirurgia) e tentava-se selecionar pelo menos um controle. Caso houvesse mais de uma acompanhante dentro dos critérios do estudo, recrutavase a de mais idade. No caso de insucesso, buscava-se na enfermaria vizinha.

\section{Modelo conceitual hierárquico e} variáveis estudadas

Postulando-se que existe uma relação de influências entre as covariáveis, a exposição de interesse e o desfecho em estudo, o trabalho foi desenvolvido tendo como base um modelo teórico conceitual estruturado em níveis hierárquicos 22 , esquematicamente apresentado na Figura 1.
Com base na literatura, algumas variáveis foram selecionadas para serem testadas em relação à capacidade de representar as dimensões e/ou níveis do modelo hierárquico. Baseados no fato de que as desigualdades sociais são condições consistentemente relacionadas ao risco de câncer do colo de útero 23, quatro variáveis, explicitadas a seguir, foram examinadas para a composição do nível distal. Para avaliar a dimensão socioeconômica, foram testadas as seguintes variáveis: anos de estudo (nenhum versus 1 ano ou mais); renda familiar percebida mensalmente em salários mínimos (SM) (sem renda/renda < 2 SM versus 2 ou mais SM); situação conjugal analisada comparando as mulheres casadas ou que viviam com companheiro, com as demais que referiram ser solteiras, viúvas ou separadas/ divorciadas (casadas versus não casadas). A variável cor da pele foi testada dicotomizada (não branca versus branca) para compor a dimensão demográfica, junto aos aspectos socioeconômicos no nível distal, levando-se em conta a autoclassificação em branca, parda ou negra fornecida pela mulher.

Alguns fatores de risco de câncer do colo de útero foram examinados para representar o nível intermediário. Na dimensão sexual/reprodutiva foram consideradas as seguintes variáveis: idade da menarca (variável contínua); idade da primei-

Figura 1

Modelo teórico conceitual.

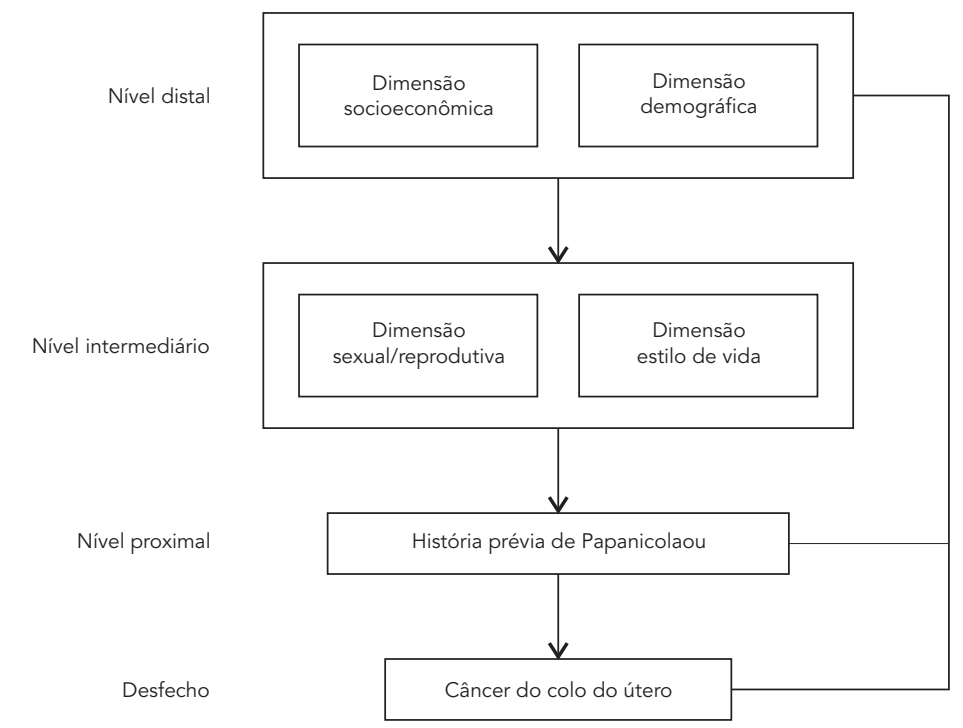


ra relação sexual (avaliada em quartis); idade da primeira gestação ( $<20$ e $\geq 20$ anos); paridade (nulípara ou 1 filho; 2 ou 3 ; 4 ou 5 e 6 ou mais filhos); número de parceiros sexuais ao longo da vida (2 e mais parceiros versus 1 parceiro); antecedente de aborto (sim/não) e antecedente de parto domiciliar (sim/não). Postulando-se que os hábitos das pessoas são determinados pelas condições socioeconômicas e demográficas, o uso de pílula anticoncepcional na vida ( $\operatorname{sim} /$ não) e o uso de tabaco (sim/não) foram examinados no contexto do estilo de vida, compondo, também, o nível intermediário. Foi considerada usuária de tabaco quem informou fumar ou ter fumado, em média, um cigarro - ou equivalente em charuto ou cachimbo - diariamente, pelo menos por um ano.

Os pontos de corte para variáveis ordinais foram definidos tendo como base a literatura e/ou a avaliação da linearidade da variação do logaritmo da chance do desfecho em relação aos níveis propostos para tais variáveis.

Com a expectativa de determinar o efeito do teste de Papanicolaou no desfecho câncer do colo de útero, controlando o potencial confundimento dos representantes dos níveis superiores do modelo teórico conceitual, a história prévia de teste de Papanicolaou, exposição principal deste estudo, compôs o nível proximal, sendo os históricos de exames realizados além dos 36 meses da data da entrevista/diagnóstico, distribuídos em três categorias (nenhum; 1 ou 2 e 3 ou mais exames).

\section{Instrumentos e coleta de dados}

Após a formulação do convite, leitura e assinatura do consentimento informado, a coleta de informações foi realizada por uma das pesquisadoras (M.I.N.) pelo uso de um questionário padronizado em blocos que abordava aspectos sociodemográficos, sexuais e reprodutivos, de estilo de vida e de acesso ao teste de Papanicolaou. O último bloco foi previamente utilizado em inquérito conduzido no Brasil pelo Instituto Nacional de Câncer 19, e três das questões nele contidas nortearam as definições de história de realização do teste de Papanicolaou utilizadas no presente estudo.

Com a pergunta "A Senhora, alguma vez, fez exame preventivo?”, foi definido o acesso ao teste de Papanicolaou na vida (sim/não). Tomando-se como referência o ponto de corte de 36 meses da entrevista 24; e as questões: "Em toda a sua vida, quantos exames preventivos a Senhora fez?" e "Quantos exames preventivos a Senhora fez nos últimos três anos?” foram delimitadas a história recente (número de exames realizados nos últi- mos 36 meses da entrevista/diagnóstico, distribuídos nas categorias nenhum; 1 ou 2 e 3 ou mais exames) e a história prévia (número de exames realizados além dos 36 meses da entrevista/diagnóstico distribuídos nas categorias nenhum; 1 ou 2 e 3 ou mais exames). Uma questão sobre a realização do último exame foi usada para classificar se ele foi feito por rotina (sim/não) e outra se ele foi feito em unidade do SUS (sim/não).

A entrevista ocorreu em local reservado, de modo a garantir o anonimato e a confidencialidade das informações coletadas. Alguns procedimentos estabelecidos para os casos (realização de biópsia, exames laboratoriais e estadiamento clínico) seguiram o protocolo do hospital. As coletas de material biológico específicas do estudo foram feitas imediatamente após a entrevista.

Tendo em vista a importância de distinguir a coleta do esfregaço citológico de outros exames pélvicos 12 , um esclarecimento era fornecido antes de formular as perguntas sobre o teste de Papanicolaou.

\section{Análise estatística}

Empregou-se a regressão logística não condicional para estimar as medidas de efeito ${ }^{17}$. Primeiramente, foram calculadas as OR brutas, sendo consideradas para análises posteriores as variáveis com valor de $\mathrm{p} \leq 0,2525$. A seguir, a modelagem foi implementada e foram obtidas OR ajustadas por variáveis da mesma dimensão e/ou nível hierárquico. As variáveis que tiveram valor de $\mathrm{p} \leq 0,25$ ou aquelas que, quando retiradas do modelo provocaram uma variação de pelo menos $10 \%$ na OR de outras variáveis da mesma dimensão ou nível em construção, foram selecionadas como fator de confusão e incluídas na análise multivariada.

Tendo como base o modelo teórico conceitual, as variáveis remanescentes foram testadas na modelagem hierárquica, começando pelo nível distal, obtendo-se OR ajustadas pelas variáveis do mesmo nível e do nível superior e não para os possíveis fatores de mediação ${ }^{22}$. As variáveis remanescentes características da dimensão estilo de vida foram testadas no nível intermediário da modelagem hierárquica depois da avaliação das representantes distais e da dimensão sexual/ reprodutiva. A composição do modelo final foi baseada no critério do valor de $\mathrm{p}<0,1026$ e na variação das estimativas de OR após a retirada do suposto fator de confusão do modelo, usando o ponto de corte arbitrado em pelo menos $10 \% 27$. Adicionalmente, independente de significância estatística, fatores considerados biologicamente plausíveis de determinar o aparecimento do desfecho em estudo foram incluídos no modelo 
final 26 . Todas as análises foram ajustadas pelas variáveis de pareamento 17 .

A idade entrou na modelagem como variável contínua e os municípios de residência foram agregados por proximidade geográfica como descrito previamente, sendo a categoria de referência formada por Nova Iguaçu, Duque de Caxias e Magé. Potenciais interações multiplicativas entre a história prévia de teste de Papanicolaou e outras variáveis do modelo final foram avaliadas pela aplicação do teste de razão de verossimilhança. $\mathrm{O}$ ajuste do modelo final foi testado com o uso da estatística de Hosmer-Lemeshow ${ }^{28}$. Os dados foram analisados com o uso do programa estatístico Stata 10.0 (Stata Corp. College Station, Estados Unidos).

\section{Aspectos éticos}

O estudo foi aprovado pelos comitês de ética do HGNI (parecer no. 013/2006) e da ENSP/Fiocruz (parecer $n^{\circ}$. 109/2009) e seguiu rigorosamente todos os devidos cuidados na sua condução.

\section{Resultados}

No período de estudo, 169 mulheres com diagnóstico histopatológico de câncer do colo de útero atendidas no HGNI preencheram os critérios de inclusão. Foram excluídos sete $(4,1 \%)$ casos, sendo cinco internados em fase terminal e dois por problemas de saúde mental impeditivos de responder às perguntas do questionário. Ocorreram dez perdas $(5,9 \%)$, correspondendo a sete casos que foram a óbito antes da entrevista e três recusas. Dos 179 controles selecionados, foram excluídos quatro $(2,2 \%)$ com antecedentes de histerectomia total e foram perdidas seis $(3,4 \%)$. Das participantes do grupo controle efetivamente entrevistadas (169), 54 (32\%) foram recrutadas na Clínica Médica, 32 (18,9\%) na Cirurgia Geral, $32(18,9 \%)$ na Ortopedia, $32(18,9 \%)$ na Ginecologia, 11 (6,5\%) na Maternidade e 7 (4,1\%) na Neurocirurgia. Houve perda dessa informação para 1 controle $(0,6 \%)$. Assim, participaram do estudo 321 mulheres, sendo 152 casos e 169 controles. O percentual de casos que apresentou doença invasora e microinvasora foi de $94,1 \%$ e $5,9 \%$, respectivamente. Predominou a histopatologia de carcinoma de células escamosas $(90,1 \%)$, seguida de adenocarcinoma (7,9\%), carcinoma adenoescamoso $(1,3 \%)$ e carcinoma indiferenciado $(0,7 \%)$

Os casos tinham idade entre 25 e 68 anos (média de 47,3 anos e desvio padrão - $\mathrm{DP}=10,3$ ) e a dos controles variou de 25-67 anos (média de 46,6 anos; $\mathrm{DP}=10,8$ ). A média de tempo de residên- cia na Baixada Fluminense foi de 33,2 anos (variação: 5-64 anos) e de 36,2 anos (variação: 3-67 anos) para casos e controles, respectivamente. A metade dos casos e dos controles era residente do Município de Nova Iguaçu. A comparação de casos e controles segundo o município de residência agregado por proximidade geográfica não mostrou diferença estatisticamente significativa.

A média de anos de estudo dos casos (4,4 anos; DP $=3,4$ anos) foi inferior a dos controles (5,8 anos; $\mathrm{DP}=3,6$ anos), com valor de $\mathrm{p}<0,001$ e apenas uma participante (controle) referiu mais de 12 anos de estudo. Os casos tiveram a média de idade da menarca $(13,0$ anos; $\mathrm{DP}=1,9)$ ligeiramente superior a dos controles (12,8 anos; $\mathrm{DP}=1,8)$, com diferença estatisticamente não significativa. A Tabela 1 mostra a distribuição das variáveis categóricas características da população de estudo selecionadas segundo níveis hierárquicos.

Verificou-se que a maioria $(92,2 \%)$ das mulheres fez pelo menos um teste de Papanicolaou na vida. A contagem de exames variou de 0-30. Cerca de 30\% delas estavam há mais de 36 meses sem realizar o teste. Relacionando as histórias de exames realizados e o câncer do colo de útero, foram encontradas diferenças estatisticamente significativas nas proporções, sugerindo que os grupos diferem segundo os padrões de acesso e o motivo que levou à realização do último exame reportado (Tabela 2).

$\mathrm{Na}$ análise univariada, a renda familiar (valor de $\mathrm{p}=0,694$ ) e a idade da menarca (valor de $\mathrm{p}=0,363$ ) não foram incluídas no modelo multivariado. Foi encontrada uma associação inversa com tendência linear (valor de $\mathrm{p}<0,0001$ ) entre a idade da primeira relação e câncer do colo de útero. Na análise intrabloco, o antecedente de aborto (valor de $\mathrm{p}=0,905$ ), o parto domiciliar (valor de $\mathrm{p}=0,789$ ) e a idade da primeira gestação (valor de $\mathrm{p}=0,434$ ) perderam significância estatística no ajustamento da dimensão sexual/ reprodutiva e na população estudada não constituíram fatores de confusão. A Tabela 3 mostra os resultados das análises brutas e ajustadas por dimensão/nível hierárquico.

Na modelagem hierárquica, as variáveis remanescentes (cor da pele, anos de estudo, situação conjugal, idade da primeira relação, paridade, parceiros sexuais na vida, uso de anticoncepcional e uso de tabaco) foram testadas como potenciais fatores de confusão. As variáveis: cor da pele (valor de $\mathrm{p}=0,107$ ) e uso de anticoncepcional (valor de $\mathrm{p}=0,148$ ) foram eliminadas nessa etapa da análise. Entretanto, ter história prévia de Papanicolaou de três ou mais exames comparado com nenhum, status marital de casada/unida, início da vida sexual tardio (20-31 anos versus 
Distribuição das variáveis categóricas candidatas à composição dos níveis do modelo hierárquico: estudo caso-controle de câncer do colo do útero. Baixada Fluminense, Rio de Janeiro, Brasil, 2007-2010.

\begin{tabular}{|c|c|c|c|c|}
\hline Nível/Dimensão/Variável & $\begin{array}{c}\text { Total } \\
(\mathrm{N}=321) \\
\text { Total }(\%)\end{array}$ & $\begin{array}{c}\text { Casos } \\
(n=152) \\
n(\%)\end{array}$ & $\begin{array}{l}\text { Controles } \\
\begin{array}{c}(n=169) \\
n(\%)\end{array}\end{array}$ & Valor de $\mathrm{p}$ \\
\hline \multicolumn{5}{|l|}{ Nível distal } \\
\hline \multicolumn{5}{|l|}{ Dimensão socioeconômica } \\
\hline Anos de estudo & & & & 0,007 \\
\hline Nenhum & $42(13,1)$ & $28(18,4)$ & $14(8,3)$ & \\
\hline $1 \mathrm{ou}+$ & $279(86,9)$ & $124(81,6)$ & $155(91,7)$ & \\
\hline Renda familiar (em salários mínimos) & & & & 0,728 \\
\hline Sem renda $a<2$ & $218(68,1)$ & $105(69,1)$ & $113(67,3)$ & \\
\hline $2 \mathrm{ou}+$ & $102(31,9)$ & $47(30,9)$ & $55(32,7)$ & \\
\hline Situação conjugal (casada/unida) & & & & 0,019 \\
\hline Sim & $207(64,5)$ & $88(57,9)$ & $119(70,4)$ & \\
\hline Não & $114(35,5)$ & $64(42,1)$ & $50(29,6)$ & \\
\hline \multicolumn{5}{|l|}{ Dimensão demográfica } \\
\hline Cor da pele autorreferida & & & & 0,176 \\
\hline Não branca & $225(70,1)$ & $101(66,5)$ & $124(73,4)$ & \\
\hline Branca & $96(29,9)$ & $51(33,5)$ & $45(26,6)$ & \\
\hline \multicolumn{5}{|l|}{ Nível intermediário } \\
\hline \multicolumn{5}{|l|}{ Dimensão sexual/reprodutiva } \\
\hline Idade da primeira relação sexual * & & & & $<0,0001$ \\
\hline Q1 & $87(27,3)$ & $54(35,5)$ & $33(19,8)$ & \\
\hline Q2 & $82(25,7)$ & $42(27,6)$ & $40(23,9)$ & \\
\hline Q3 & $79(24,8)$ & $37(24,3)$ & $42(25,2)$ & \\
\hline Q4 & $71(22,2)$ & $19(12,6)$ & $52(31,1)$ & \\
\hline Idade da primeira gestação (anos) ** & & & & 0,001 \\
\hline$<20$ & $174(54,4)$ & $98(64,5)$ & $76(45,2)$ & \\
\hline$\geq 20$ & $146(45,6)$ & $54(35,5)$ & $92(54,8)$ & \\
\hline Paridade & & & & $<0,001$ \\
\hline Nenhum ou 1 & $48(15,0)$ & $13(8,6)$ & $35(20,7)$ & \\
\hline 2 ou 3 & $141(43,9)$ & $59(38,8)$ & $82(48,5)$ & \\
\hline 4 ou 5 & $70(21,8)$ & $40(26,3)$ & $30(17,8)$ & \\
\hline 6 ou mais & $62(19,3)$ & $40(26,3)$ & $22(13,0)$ & \\
\hline Número de parceiros sexuais na vida & & & & $<0,0001$ \\
\hline $2 \mathrm{ou}+$ & $236(73,5)$ & $126(82,9)$ & $110(65,1)$ & \\
\hline 1 & $85(26,5)$ & $26(17,1)$ & $59(34,9)$ & \\
\hline Antecedente de aborto & & & & 0,191 \\
\hline Sim & $119(37,1)$ & $62(40,8)$ & $57(33,7)$ & \\
\hline Não & $202(62,9)$ & $90(59,2)$ & $112(66,3)$ & \\
\hline Parto domiciliar & & & & 0,058 \\
\hline Sim & $58(18,1)$ & $34(22,4)$ & $24(14,2)$ & \\
\hline Não & $263(81,9)$ & $118(77,6)$ & $145(85,8)$ & \\
\hline \multicolumn{5}{|l|}{ Dimensão estilo de vida } \\
\hline Uso de anticoncepcional & & & & 0,014 \\
\hline Sim & $234(72,9)$ & $101(66,5)$ & $133(78,7)$ & \\
\hline Não & $87(27,1)$ & $51(33,5)$ & $36(21,3)$ & \\
\hline Uso de tabaco & & & & 0,126 \\
\hline Sim & $144(44,9)$ & $75(49,3)$ & $69(40,8)$ & \\
\hline Não & $177(55,1)$ & $77(50,7)$ & $100(59,2)$ & \\
\hline
\end{tabular}

* Quartil 1 (referência): 10-15 anos; quartil 2: 16-17 anos; quartil 3: 18-19 anos; quartil 4: 20-31 anos;

** Análise restrita a 320 observações. 
Padrão de acesso e uso do teste de Papanicolaou: estudo caso-controle de câncer do colo do útero. Baixada Fluminense Rio de Janeiro, Brasil, 2007-2010.

\begin{tabular}{|c|c|c|c|}
\hline Variável & $\begin{array}{c}\text { Casos } \\
\begin{array}{c}(n=152) \\
n(\%)\end{array}\end{array}$ & $\begin{array}{l}\text { Controles } \\
\begin{array}{c}(n=169) \\
n(\%)\end{array}\end{array}$ & Valor de $p$ * \\
\hline \multicolumn{4}{|c|}{ Papanicolaou alguma vez na vida } \\
\hline Sim & $136(89,5)$ & $160(94,7)$ & 0,083 \\
\hline Não & $16(10,5)$ & $9(5,3)$ & \\
\hline \multicolumn{4}{|c|}{ História recente de Papanicolaou (dentro 36 meses) } \\
\hline $\operatorname{Sim}$ & $110(72,4)$ & $112(66,3)$ & 0,238 \\
\hline Não & $42(27,6)$ & $57(33,7)$ & \\
\hline Nenhum & $42(27,6)$ & $57(33,7)$ & 0,018 \\
\hline 1 ou 2 exames & $87(57,2)$ & $71(42,0)$ & \\
\hline 3-5 exames & $23(15,1)$ & $41(24,3)$ & \\
\hline \multicolumn{4}{|c|}{ História prévia de Papanicolaou (além de 36 meses) } \\
\hline Sim & $116(76,3)$ & $157(92,9)$ & $<0,0001$ \\
\hline \multirow[t]{2}{*}{ Não } & $36(23,7)$ & $12(7,1)$ & \\
\hline & $36(23,7)$ & $12(7,1)$ & $<0,0001$ \\
\hline \multicolumn{4}{|l|}{ Nenhum } \\
\hline 1 ou 2 exames & $49(32,2)$ & $28(16,6)$ & \\
\hline 3 ou mais & $67(44,0)$ & $129(76,3)$ & \\
\hline \multicolumn{4}{|c|}{$p$ de tendência $\leq 0,0001$} \\
\hline \multicolumn{4}{|c|}{ Último Papanicolaou de rotina ** } \\
\hline $\operatorname{Sim}$ & $55(40,4)$ & $119(74,4)$ & $<0,0001$ \\
\hline Não & $81(59,6)$ & $41(25,6)$ & \\
\hline \multicolumn{4}{|c|}{ Último Papanicolaou no SUS ** } \\
\hline Sim & $106(77,9)$ & $113(70,6)$ & 0,153 \\
\hline Não & $30(22,1)$ & $47(29,4)$ & \\
\hline
\end{tabular}

* Valor de p com nível de significância < 0,05;

** Corresponde a 296 observações com acesso ao Papanicolaou.

10-15 anos), mais de um filho e mais de um parceiro sexual na vida permaneceram estatisticamente associadas ao câncer do colo de útero. Depois do ajustamento, optou-se por manter as variáveis anos de estudo (OR = 1,03; IC95\%: 0,433; 2,468) e uso do tabaco (OR = 0,95; IC95\%: 0,560; $1,629)$ no modelo final como fatores de confusão da relação história prévia de Papanicolaou e câncer do colo de útero (Tabela 4).

A inclusão dos termos multiplicativos não evidenciou modificação de efeito na relação do histórico de teste de Papanicolaou e câncer do colo de útero. De acordo com a estatística Hosmer-Lemeshow, o modelo foi adequadamente ajustado (valor de $\mathrm{p}=0,1951$ ).

\section{Discussão}

A grande contribuição do presente estudo foi ter mostrado que a história prévia de exames preventivos realizados além dos últimos três anos que precederam o diagnóstico conferiu uma proteção de mais de $80 \%$ no aparecimento do câncer do colo do útero. Além disso, ter mais de um parceiro ao longo da vida e mais de um filho aumentam o risco; ao passo que ser casada e ter vida sexual iniciada mais tardiamente reduzem o risco de doença.

Verificou-se que nos municípios da Baixada Fluminense, em que pesem as ações implementadas para controle do câncer do colo de útero, ainda há mulheres sem acesso ao rastreamento ou com exames atrasados. Ao mesmo tempo, existem outras que fizeram mais exames do que 
Tabela 3

Associação das variáveis candidatas e câncer do colo do útero: odds ratios brutas e ajustadas pelas exposições do mesmo nível hierárquico: estudo casocontrole de câncer do colo do útero. Baixada Fluminense, Rio de Janeiro, Brasil, 2007-2010.

\begin{tabular}{|c|c|c|c|c|c|c|}
\hline Nível/Dimensão/Variável & $\mathrm{OR}_{\text {bruta }}$ & Valor de p & IC95\% & OR ajustada * & Valor de $p$ ** & IC95\% \\
\hline \multicolumn{7}{|l|}{ Nível distal } \\
\hline \multicolumn{7}{|c|}{ Dimensão socioeconômica } \\
\hline \multicolumn{7}{|l|}{ Anos de estudo } \\
\hline$\geq 1$ & 1,00 & & & 1,00 & & \\
\hline Nenhum & 2,52 & 0,011 & 1,$235 ; 5,151$ & 2,66 & 0,008 & 1,$292 ; 5,513$ \\
\hline \multicolumn{7}{|c|}{ Renda familiar (em salários mínimos) } \\
\hline$\geq 2$ & 1,00 & & & - & - & - \\
\hline Sem renda a $<2$ & 1,09 & 0,694 & 0,$684 ; 1,767$ & & & \\
\hline \multicolumn{7}{|c|}{ Situação conjugal (casada/unida) } \\
\hline Não & 1,00 & & & 1,00 & & \\
\hline Sim & 0,58 & 0,026 & 0,$366 ; 0,937$ & 0,55 & 0,017 & 0,$344 ; 0,899$ \\
\hline \multicolumn{7}{|l|}{ Dimensão demográfica } \\
\hline \multicolumn{7}{|l|}{ Cor da pele autorreferida } \\
\hline Branca & 1,00 & & & 1,00 & & \\
\hline Não branca & 0,71 & 0,171 & 0,$441 ; 1,155$ & 0,66 & 0,107 & 0,$407 ; 1,091$ \\
\hline \multicolumn{7}{|l|}{ Nível intermediário } \\
\hline \multicolumn{7}{|c|}{ Dimensão sexual/reprodutiva } \\
\hline Idade da menarca & 1,05 & 0,363 & 0,$937 ; 1,191$ & - & - & - \\
\hline \multicolumn{7}{|c|}{ 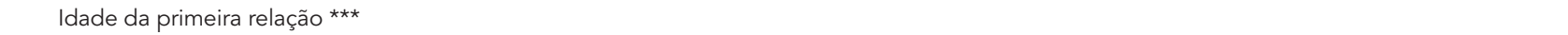 } \\
\hline Q1 & 1,00 & & & 1,00 & & \\
\hline Q2 & 0,60 & 0,116 & 0,$324 ; 1,131$ & 0,76 & 0,409 & 0,$396 ; 1,457$ \\
\hline Q3 & 0,50 & 0,034 & 0,$270 ; 0,948$ & 0,63 & 0,180 & 0,$330 ; 1,230$ \\
\hline Q4 & 0,19 & 0,000 & 0,$094 ; 0,390$ & 0,34 & 0,007 & 0,$157 ; 0,741$ \\
\hline$p$ de tendência & & $<0,0001$ & & & 0,008 & \\
\hline \multicolumn{7}{|c|}{ Idade da primeira gestação (anos) } \\
\hline$\geq 20$ & 1,00 & & & 1,00 & & \\
\hline$<20$ & 2,35 & $<0,001$ & 1,$477 ; 3,753$ & 1,28 & 0,434 & 0,$684 ; 2,422$ \\
\hline \multicolumn{7}{|l|}{ Paridade } \\
\hline Nenhum ou 1 & 1,00 & & & 1,00 & & \\
\hline 2 ou 3 & 1,96 & 0,067 & 0,$953 ; 4,038$ & 1,45 & 0,334 & 0,$680 ; 3,106$ \\
\hline 4 ou 5 & 3,74 & 0,001 & 1,$676 ; 8,352$ & 2,77 & 0,018 & 1,$189 ; 6,490$ \\
\hline $6 \mathrm{ou}+$ & 5,32 & 0,000 & 2,$261 ; 12,531$ & 3,00 & 0,020 & 1,$188 ; 7,597$ \\
\hline \multicolumn{7}{|c|}{ Parceiros sexuais na vida } \\
\hline 1 & 1,00 & & & 1,00 & & \\
\hline $2 \mathrm{ou}+$ & 2,80 & 0,000 & 1,$632 ; 4,810$ & 2,41 & 0,003 & 1,$348 ; 4,320$ \\
\hline \multicolumn{7}{|c|}{ Antecedente de aborto } \\
\hline Não & 1,00 & & & 1,00 & & \\
\hline $\operatorname{Sim}$ & 1,34 & 0,211 & 0,$845 ; 2,143$ & 0,96 & 0,905 & 0,$576 ; 1,627$ \\
\hline \multicolumn{7}{|l|}{ Parto domiciliar } \\
\hline Não & 1,00 & & & 1,00 & & \\
\hline $\operatorname{Sim}$ & 1,73 & 0,081 & 0,$933 ; 3,216$ & 0,90 & 0,789 & 0,$430 ; 1,896$ \\
\hline \multicolumn{7}{|l|}{ Dimensão estilo de vida } \\
\hline \multicolumn{7}{|l|}{ Hábito de fumar } \\
\hline Não & 1,00 & & & 1,00 & & \\
\hline Sim & 1,43 & 0,12 & 0,$910 ; 2,253$ & 1,49 & 0,087 & 0,$943 ; 2,365$ \\
\hline
\end{tabular}

(continua) 
Tabela 3

\begin{tabular}{|c|c|c|c|c|c|c|}
\hline Nível/Dimensão/Variável & OR $R_{\text {bruta }}$ & Valor de $\mathrm{p}$ & IC95\% & OR ajustada $^{*}$ & Valor de $p$ ** & IC95\% \\
\hline \multicolumn{7}{|c|}{ Uso de anticoncepcional na vida } \\
\hline Não & 1,00 & & & 1,00 & & \\
\hline Sim & 0,54 & 0,020 & 0,$329 ; 0,908$ & 0,52 & 0,015 & 0,$317 ; 0,883$ \\
\hline \multicolumn{7}{|l|}{ Nível proximal } \\
\hline \multicolumn{7}{|c|}{ Papanicolaou além de 36 meses } \\
\hline Nenhum exame & 1,00 & & & & & \\
\hline 1 ou 2 exames & 0,58 & 0,195 & 0,$261 ; 1,314$ & - & - & - \\
\hline 3 ou mais & 0,17 & 0,000 & 0,$084 ; 0,359$ & & & \\
\hline
\end{tabular}

IC95\%: intervalo de 95\% de confiança.

* Ajustadas pelas variáveis da dimensão/nível hierárquico e de pareamento;

** Valor de p com nível de significância <0,25;

*** Quartil 1: 10-15 anos; quartil 2: 16 e 17 anos; quartil 3: 18 e 19 anos; quartil 4: 20-31 anos;

Nota: os traços (-) representam as categorias não aplicáveis.

Tabela 4

Modelo final de história prévia de realização de teste de Papanicolaou e câncer do colo do útero: estudo caso-controle. Baixada Fluminense, Rio de Janeiro, Brasil, 2007-2010.

\begin{tabular}{|c|c|c|}
\hline \multirow[t]{2}{*}{ Nível/Variável } & \multicolumn{2}{|c|}{ Modelo Final } \\
\hline & $\mathrm{OR}_{\text {ajustada }}$ & IC95\% \\
\hline \multicolumn{3}{|l|}{ Nível distal } \\
\hline \multicolumn{3}{|l|}{ Situação conjugal } \\
\hline Casada/Unida & 0,49 & 0,$276 ; 0,859$ \\
\hline \multicolumn{3}{|l|}{ Nível intermediário * } \\
\hline \multicolumn{3}{|l|}{ Idade da primeira relação ** } \\
\hline Q1 & 1,00 & \\
\hline Q2 & 0,78 & 0,$386 ; 1,599$ \\
\hline Q3 & 0,59 & 0,$289 ; 1,217$ \\
\hline Q4 & 0,31 & 0,$130 ; 0,725$ \\
\hline \multicolumn{3}{|l|}{$\mathrm{p}$ de tendência $=0,006$} \\
\hline \multicolumn{3}{|c|}{ Número de parceiros sexuais } \\
\hline $2 \mathrm{ou}+$ & 2,08 & 1,$095 ; 3,974$ \\
\hline \multicolumn{3}{|l|}{ Paridade } \\
\hline Nenhum ou 1 & 1,00 & \\
\hline 2 ou 3 & 2,51 & 1,$074 ; 5,875$ \\
\hline 4 ou 5 & 4,12 & 1,$591 ; 10,332$ \\
\hline 6 filhos ou mais & 3,68 & 1,$313 ; 10,332$ \\
\hline \multicolumn{3}{|l|}{$p$ de tendência $=0,011$} \\
\hline \multicolumn{3}{|l|}{ 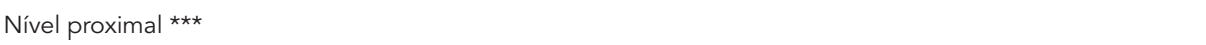 } \\
\hline \multicolumn{3}{|c|}{ Papanicolaou além de 36 meses } \\
\hline Nenhum exame & 1,00 & \\
\hline 1 ou 2 exames & 0,67 & 0,$275 ; 1,640$ \\
\hline 3 exames ou mais & 0,16 & 0,$074 ; 0,384$ \\
\hline $\mathrm{p}$ de tendência $<0,0001$ & & \\
\hline
\end{tabular}

IC95\%: intervalo de 95\% de confiança.

* Ajustada pelo nível distal (incluindo anos de estudo) e pelas variáveis de pareamento (idade e município de residência);

** Quartil 1: 10-15 anos; quartil 2: 16-17 anos; quartil 3: 18-19 anos; quartil 4: 20-31 anos;

*** Ajustada pelos níveis distal e intermediário (incluindo uso de tabaco) e pelas variáveis de pareamento (idade e município de residência). 
seria esperado, possivelmente em reflexo à estratégia de rastreio do tipo demanda espontânea.

Observando o padrão de acesso, o estudo mostrou que as participantes tinham história recente (dentro dos últimos 36 meses) de até cinco exames, circunstâncias que estão sinalizando para a distribuição irregular de exames 29 , para a sobrecarga do sistema 30 e para o consumo desnecessário de recursos 31. Também, chamou a atenção o fato de que a proporção de casos que fizeram um ou dois exames foi maior que a dos controles no período referido. Tais achados podem ter relação com as necessidades percebidas pelo profissional de saúde ou pela paciente. De um lado, existe a possibilidade de o profissional solicitar o exame com finalidade diagnóstica, embora ele seja eminentemente de rastreio 29 . Por outro lado, diante da necessidade, também é a paciente que, por razões pessoais, toma a decisão de buscar o cuidado a fim de fazer uso de algum recurso 32 . Às vezes, o exame é repetido para confirmar um resultado que foi, em princípio, questionado. Porém, são as queixas ginecológicas que frequentemente motivam a busca pelo exame 29 . O último parece ser um comportamento característico do grupo de maior risco de desenvolver a doença. No presente estudo, também foi encontrada uma grande diferença entre casos e controles que tomaram o último exame de modo rotineiro.

No México, os resultados de um estudo caso-controle mostraram que $43 \%$ das mulheres participantes de rastreamento reportaram pelo menos um sintoma ${ }^{33}$. Outro estudo, também realizado no México 15, evidenciou a OR de 0,38 (IC95\%: 0,28; 0,52), ao comparar o relato de ter e não ter se submetido ao teste, desconsiderando os exames realizados nos 12 meses precedentes à entrevista, assumindo que eles tinham sido feitos com finalidade diagnóstica. Mas, sem distinguir o propósito do exame, o efeito protetor foi atenuado (OR = 0,76; IC95\%: 0,59; 0,98), sugerindo que a inclusão indiscriminada de exames realizados com finalidade de rastreio e/ou diagnóstica pode subestimar a magnitude da OR.

Assim, para se estimar o efeito de um teste de rastreamento com base nas pacientes com câncer, a possibilidade de distorção nas medidas produzidas pelo viés de autosseleção das mulheres que se apresentam para se submeter aos exames não deve ser negligenciada na seleção dos casos 34 , bem como na dos controles 31 , ainda que a propensão para realizá-los seja diferente entre as mulheres.

No atual estudo, a redução de risco de desenvolver câncer do colo de útero foi determinada tomando o cuidado de não incluir na análise os exames recentes que não permitiriam separar adequadamente casos de doença já iniciados de mulheres não doentes. Ampliando o intervalo para 36 meses e mantendo o enfoque na história prévia, a efetividade do teste de Papanicolaou foi evidenciada com a redução na estimativa de risco de câncer do colo de útero de $84 \%$ para as mulheres que fizeram três exames ou mais comparando com nenhum, depois do ajustamento do modelo hierárquico. Ainda que os diferentes critérios de definição do padrão de uso do teste de Papanicolaou dificultem a comparabilidade entre os estudos, os atuais resultados são consistentes com os encontrados na Coreia do Sul 6, que mostraram o risco relativo de 0,16 (IC95\%: 0,05; 0,46) para dois e mais exames comparado com nenhum. Também no estudo realizado na cidade de São Paulo 12, a OR foi de 0,20 (IC95\%: 0,06; 0,62) para história de três a cinco exames e de 0,12 (IC95\%: $0,03 ; 0,41)$ quando se referiu seis exames ou mais, comparando com nenhum exame.

Vale assinalar que o principal lugar referido como realização do último teste de Papanicolaou foi dentro do SUS. Especulando-se que, na população estudada, os exames anteriores tenham sido feitos em tais condições, os achados do estudo podem estar refletindo um cenário usual de assistência de uma significativa parcela de mulheres brasileiras, uma vez que, a maior fonte de pagamento do teste de Papanicolaou, em todas as regiões do Brasil, tem sido o SUS 11. Considerando que casos e controles tiveram história recente (dentro de 36 meses) de até cinco exames e que a história prévia (além de 36 meses) de um ou dois exames repercutiu em efeito protetor de câncer do colo de útero estatisticamente não significativo, cabe, ainda, interrogar se tais achados seriam ou não um reflexo da qualidade do processo que envolveu a elaboração desses exames.

Além da contribuição do teste de Papanicolaou, o estudo mostrou a associação estatisticamente significativa do número de parceiros sexuais e da paridade com o câncer do colo de útero, como tem sido reportado em outros estudos epidemiológicos 15,35. Também foi encontrado um efeito dose-resposta da variável idade da primeira relação, sendo o risco de câncer do colo de útero três vezes menor para o início da vida sexual mais tardio (20-31 anos) em relação ao mais precoce (10-15 anos), ajustado pelas outras variáveis do modelo. Das variáveis socioeconômicas e demográficas, apenas a situação conjugal permaneceu com significância estatística no modelo final, com as mulheres casadas apresentando risco menor de ter a doença.

À semelhança de outros estudos 5,7, o controle do confundimento de condições socioambientais foi feito implementando o pareamento por frequência de entrada de casos por faixa 
etária e lugar de residência. Esse procedimento pode ter tornado a população estudada de tal forma homogênea, que algumas características pessoais como cor da pele não branca e a baixa renda familiar não configuraram fatores estatisticamente associados ao câncer do colo de útero. Todavia, tendo em vista que o nível de educação é um marcador importante das condições socioeconômicas 35 , vale ressaltar que, no presente estudo, a associação da baixa escolaridade, inicialmente forte, desapareceu depois da introdução da história prévia de teste de Papanicolaou na análise multivariada, achado também encontrado no México 36. Por mais que se tenha optado por manter a variável no modelo como fator de confusão, a suspeita é que o papel da baixa escolaridade possa estar sendo mediado pelo melhor acesso ao teste de Papanicolaou na região, interpretação que, para ser adequadamente avaliada requer o uso de métodos de análise estatística diferentes dos que foram aqui usados.

Este estudo tem limitações relacionadas à variável de exposição de maior interesse. Os históricos autorreportados 37 , a dificuldade de distinguir a coleta do teste de Papanicolaou de outros procedimentos ginecológicos $12 \mathrm{e}$ a tendência a expressar um comportamento socialmente aceitável, com a submissão ao exame por mais vezes e em prazos mais atualizados 38 podem conduzir a erros de classificação. Procurou-se minimizar esses problemas deixando clara a finalidade do teste, obtendo das participantes laudos escritos e desconsiderando da análise multivariada os históricos mais recentes. Uma segunda limitação deve-se ao fato que as variáveis relativas às barreiras institucionais de acesso ao teste de Papanicolaou não foram analisadas. Outra limitação foi o longo tempo de seleção das participantes. Contudo, não se conhece, durante o período, a existência de modificações na organização dos serviços da região e acredita-se que tal fato não influenciou os resultados. Por fim, o tamanho amostral não conferiu poder para adequadamente avaliar o papel do hábito de fumar no risco de câncer do colo de útero na população estudada, embora essa relação não tenha sido o foco principal do estudo.

Entre as vantagens do estudo, pode-se considerar que o enfoque no histórico de exames realizados além de 36 meses contribuiu para superar pelo menos duas questões. A primeira se refere ao viés da autosseleção previamente mencionado e relacionado às mulheres que somente procuram o exame na condição de doentes ou apresentando sintomas, fato que poderia estar mais evidente nos meses imediatamente precedentes à entrevista ou ao diagnóstico. A segunda diz respeito à possibilidade de não realização do teste de Papanicolaou no referido prazo, mas ainda em acordo com as normas brasileiras. Ou seja, algumas mulheres poderiam estar sendo adequadamente rastreadas e ter obtido dois exames negativos por dois anos consecutivos, podendo ser consideradas, relativamente dentro do prazo da realização do próximo teste, não configurando falha do programa e tampouco das mulheres. Também o fato de ter utilizado como sériereferência mulheres com teste de Papanicolaou negativo para neoplasia no momento do recrutamento, acompanhantes de pacientes internados no HGNI, usando a estratégia de pareamento por idade e município de residência dos casos parece ter adequadamente contribuído para o controle de condições socioambientais não diretamente contempladas no questionário.

Em suma, o estudo mostrou que o teste de Papanicolaou está acessível na Baixada Fluminense, no entanto a diferença na sua utilização persiste e está associada ao desenvolvimento do câncer do colo de útero, sugerindo que mais ações são necessárias no sentido de reduzir o desperdício de recursos e aumentar a aderência às normas do programa. Levando em conta, sobretudo, o efeito protetor do histórico de três ou mais exames, mais esforços são necessários para alcançar as mulheres que nunca foram rastreadas e manter no programa aquelas que já realizaram o exame. 


\section{Resumo}

Este estudo caso-controle hospitalar foi realizado de 2007 a 2010 para estimar a associação de história prévia de colpocitologia e câncer do colo do útero na Baixada Fluminense, Rio de Janeiro, Brasil. A amostra consistiu de 152 casos histologicamente confirmados e 169 controles selecionados no mesmo hospital dos casos. A análise foi feita de acordo com um modelo em três níveis hierárquicos; considerando as variáveis sociodemográficas (nível distal), sexual/reprodutivas e de estilo de vida (nível intermediário) e a história prévia de colpocitologia (nível proximal). Odds ratios (OR) e respectivos intervalos de 95\% de confiança (IC95\%) foram calculados pela regressão logística não condicional. Ter história prévia de três ou mais colpocitologias conferiu uma proteção de $84 \%(O R=0,16$; IC95\%: 0,074; 0,384) após ajuste pelas variáveis selecionadas. Os resultados acentuam a importância do exame preventivo ginecológico no risco desse câncer e fortalecem a necessidade de aumentar a aderência às normas do programa brasileiro, bem como de identificar e capturar mulheres relutantes para o rastreamento da doença.

Neoplasias do Colo do Útero; Teste de Papanicolaou; Acesso aos Serviços de Saúde

\section{Colaboradores}

M. I. Nascimento foi responsável pela coleta e análise dos dados e rascunho do manuscrito. G. A. Silva colaborou na interpretação dos resultados e na revisão crítica do texto. G. T. R. Monteiro coordenou o trabalho, supervisionou a análise estatística e participou da elaboração do artigo.

\section{Agradecimentos}

As autoras agradecem aos professores Sergio Koifman e Michael Eduardo Reichenheim pelas importantes sugestões que tornaram possível a elaboração deste artigo.

\section{Referências}

1. Safaeian M, Solomon D. Cervical cancer prevention - cervical screening: science in evolution. Obstet Gynecol Clin North Am 2007; 34:739-60.

2. Burd EM. Human papillomavirus and cervical cancer. Clin Microbiol Rev 2003; 16:1-17.

3. Sankaranarayanan R, Ferlay J. Worldwide burden of gynaecological cancer: the size of the problem. Best Pract Res Clin Obstet Gynaecol 2006; 20: 207-25.

4. Kasinpila C, Promthet S, Vatanasapt P, Sasieni P, Parkin DM. Evaluation of the nationwide cervical screening programme in Thailand: a case-control study. J Med Screen 2011; 18:147-53.

5. Decker K, Demers A, Chateau D, Musto G, Nugent Z, Lotocki R, et al. Papanicolaou test utilization and frequency of screening opportunities among women diagnosed with cervical cancer. Open Med 2009; 3:e140-7.
6. Jun JK, Choi KS, Jung KW, Lee HY, Gapstur SM, Park EC, et al. Effectiveness of an organized cervical cancer screening program in Korea: results from a cohort study. Int J Cancer 2009; 124:188-93.

7. Murillo R, Cendales R, Wiesner C, Piñeros M, Tovar S. Effectiveness of cytology-based cervical cancer screening in the Colombian health system. Biomedica 2009; 29:354-61.

8. Yang B, Morrell S, Zuo Y, Roder D, Tracey E, Jelfs P. A case-control study of the protective benefit of cervical screening against invasive cervical cancer in NSW women. Cancer Causes Control 2008; 19:569-76.

9. Screening for squamous cervical cancer: duration of low risk after negative results of cervical cytology and its implication for screening policies. IARC Working Group on evaluation of cervical cancer screening programmes. Br Med J (Clin Res Ed) 1986; 293:659-64. 
10. Instituto Nacional de Câncer. Viva Mulher: câncer do colo do útero: informações técnico-gerenciais e ações desenvolvidas. Rio de Janeiro: Instituto Nacional de Câncer; 2002.

11. Viacava F. Acesso e uso de serviços de saúde pelos brasileiros. Radis 2010; 96:12-9.

12. Eluf-Neto J, Booth M, Muñoz N, Bosch FX, Meijer CJ, Walboomers JM. Human papillomavirus and invasive cervical cancer in Brazil. Br J Cancer 1994; 69:114-9.

13. Girianelli VR, Thuler LCS, Azevedo e Silva G. Prevalência de HPV em mulheres assistidas pela Estratégia Saúde da Família na Baixada Fluminense do Estado do Rio de Janeiro. Rev Bras Ginecol Obstet 2010; 32:39-46.

14. Gustafsson L, Adami HO. Natural history of cervical neoplasia: consistent results obtained by an identification technique. Br J Cancer 1989; 60:13241.

15. Hernández-Avila M, Lazcano-Ponce EC, Ruiz PA Romieu I. Evaluation of the cervical cancer screening programme in Mexico: a population-based case-control study. Int J Epidemiol 1998; 27:370-6.

16. Weiss NS. Adjusting for screening history in epidemiologic studies of cancer: why, when, and how to do it. Am J Epidemiol 2003; 157:957-61.

17. Szklo M, Nieto FJ. Epidemiology: beyond the basics. Boston: Jones and Bartlett Publishers; 2007.

18. Programa das Nações Unidas Para o Desenvolvimento. Atlas do Desenvolvimento Humano no Brasil. Ranking do IDH-M dos municípios do Brasil. http://www.pnud.org.br/atlas/tabelas/index. php (acessado em 31/Jan/2012).

19. Instituto Nacional de Câncer. Inquérito domiciliar sobre comportamentos de risco e morbidade referida de doenças e agravos não transmissíveis: Brasil, 15 capitais e Distrito Federal, 2002-2003. Rio de Janeiro: Instituto Nacional de Câncer; 2004.

20. Organização Mundial de Saúde. Classificação estatística internacional de doenças e problemas relacionados à saúde, 10a revisão; São Paulo: EdUSP; 1997.

21. Instituto Nacional de Câncer. Diretrizes brasileiras para o rastreamento do câncer do colo do útero. Rio de Janeiro: Instituto Nacional de Câncer; 2011.

22. Victora CG, Huttly SR, Fuchs SC, Olinto MT. The role of conceptual frameworks in epidemiological analysis: a hierarchical approach. Int J Epidemiol 1997; 26:224-7.

23. Parikh S, Brennan P, Boffetta P. Meta-Analysis of social inequality and the risk of cervical cancer. Int J Cancer 2003; 105:687-91.

24. Somkin CP, McPhee SJ, Nguyen T, Stewart S, Shema SJ, Nguyen B, et al. The effect of access and satisfaction on regular mammogram and Papanicolaou test screening in a Multiethnic population. Med Care 2004; 42:914-26.
25. Mickey RM, Greenland S. The impact of confounder selection criteria on effect estimation. Am J Epidemiol 1989; 129:125-37.

26. Olinto MTA, Victora CG, Barros FC, Tomasi E. Determinantes da desnutrição infantil em uma população de baixa renda: um modelo de análise hierarquizado. Cad Saúde Pública 1993; 9 Suppl 1:S14-27.

27. Greenland S, Rothman KJ. Introduction to stratified analysis. In: Rothman KJ, Greenland S, Lash TL, editors. Modern epidemiology. 3rd Ed. Philadelphia: Lippincott Williams \& Wilkins; 2008. p. 258-82.

28. Hosmer DW, Lemeshow S. Applied logistic regression. 2nd Ed. New York: Wiley-Interscience; 2000.

29. Pinho AA, França Junior I, Schraiber LB, D’Oliveira AFPL. Cobertura e motivos para a realização ou não do teste de Papanicolaou no município de São Paulo. Cad Saúde Pública 2003; 19 Suppl 2: S303-13.

30. Quadros CAT, Victora CG, Dias-da-Costa JS. Coverage and focus of a cervical cancer prevention program in southern Brazil. Rev Panam Salud Pública 2004; 16:223-32.

31. Eluf-Neto J, Nascimento CM. Cervical cancer in Latin América. Semin Oncol 2001; 28:188-97.

32. Donabedian A. Aspects of medical care administration. Cambridge: Harvard University Press; 1973.

33. Lazcano-Ponce EC, Rojas-Martínez R, López-Acuña MP, López-Carrillo L, Hernández-Ávila M. Reproductive risk factors and uterine cervix cancer in Mexico City. Salud Pública Méx 1993; 35:65-73.

34. Weiss NS, Rossing MA. Healthy screenee bias in epidemiologic studies of cancer incidence. Epidemiology 1996; 7:319-22.

35. Franceschi S, Plummer M, Clifford G, De Sanjose S, Bosch X, Herrero R, et al. Differences in the risk of cervical cancer and human papillomavirus infection by education level. Br J Cancer 2009; 101: 865-70.

36. Jiménez-Pérez M, Thomas DB. Has the use of pap smears reduced the risk of invasive cervical cancer in Guadalajara, Mexico? Int J Cancer 1999; 82:804-9.

37. Rauscher GH, Johnson TP, Cho YI, Walk JA. Accuracy of self-reported cancer-screening histories: a meta-analysis. Cancer Epidemiol Biomarkers Prev 2008; 17:748-57.

38. McPhee SJ, Nguyen TT, Shema SJ, Nguyen B, Somkin C, Vo P, et al. Validation of recall of breast and cervical cancer screening by women in an ethnically diverse population. Prev Med 2002; 35 463-73.

Recebido em 05/Fev/2012

Versão final reapresentada em 30/Abr/2012 Aprovado em 28/Mai/2012 